\title{
EVALUATION OF EFFECT OF CMA RADIUS ON SOQPSK EQUALIZATION
}

\author{
Henry Umuolo, Habtamu Betelle and Solomon Thang \\ Faculty Advisors: \\ Dr. Arlene Cole-Rhodes and Dr. Farzad Moazzami \\ Department of Electrical and Computer Engineering \\ Morgan State University
}

\begin{abstract}
In this work we investigate the effect of using different values of the radius in a constant modulus algorithm (CMA) equalizer for shaped offset quadrature-phase shift keying (SOQPSK), which is transmitted using the iNET data packet structure. The iNET packet structure contains known data bits (the preamble and asynchronous marker (ASM) bits) within each data packet, and these are used to initialize a block processing CMA algorithm. Two real-time processing methods are investigated here, which are referred to as the 'Single block processing' and the 'Block-by-block processing' methods. Both processing methods require that a number of data packets be discarded while the CMA equalizer converges. In this work we will investigate the effect on the performance of the CMA equalizer of changing its equalizing radius, and also the effect of processing different numbers of data packets based on the same initialization. Based on the results of these tests, a final choice of parameters will be made and we will produce a bit error rate curve for a single telemetry channel.
\end{abstract}

\section{INTRODUCTION}

We investigate the performance of the constant modulus algorithm (CMA) equalizer, when using different values of the CMA radius, for which has data transmitted using shaped offset quadrature-phase shift keying (SOQPSK) modulation. Two different block processing methods are considered for real-time CMA equalization, and these methods are applied to equalize data bits, which have been transmitted over an aeronautical channel, using SOQPSK modulation in the INET packet structure. The iNET packet structure contains known data bits (the preamble and asynchronous marker (ASM) bits) within each data packet, and these are used to initialize the block processing CMA algorithms. CMA is a blind equalizer which recovers unknown transmitted data by forcing the equalized points to lie on a circle of a fixed radius (see [1]). In this work we consider the effect of varying the CMA radius on the performance of this equalizer. SOQPSK modulation is a type of continuous phase modulation (CPM) whose symbols are therefore of a constant modulus, and these actually lie on a circle of radius 1 . Previous work by Law [2] had noted some difficulty in using the CMA equalizer for this application, but in this work we evaluate two block processing methods, which are referred to as block-by-block and single block processing [3]. Both of these methods have an initialization stage which requires a number of data packets to be discarded during convergence of CMA. We will investigate the effect of using different values of radius on the convergence of the CMA cost function. The two 
block processing methods discussed here have been chosen in order to achieve real time performance and both are evaluated using the different radii.

\section{COMMUNICATION MODEL}

The communication model for our application is shown in figure 1. It shows the transmitter, receiver and channel, together with some basic operations which occur at the transmitter and at the receiver. A binary data stream is transmitted in the INET packet structure which is made up preamble (128 predefined bits), ASM (64 bits) and the actual data bits composed of 6144 randomly generated binary bits. The preamble and ASM is a training sequence, which can be used to identify the start of the data, to calculate the MMSE initializer for CMA equalization, and for error correction [4].

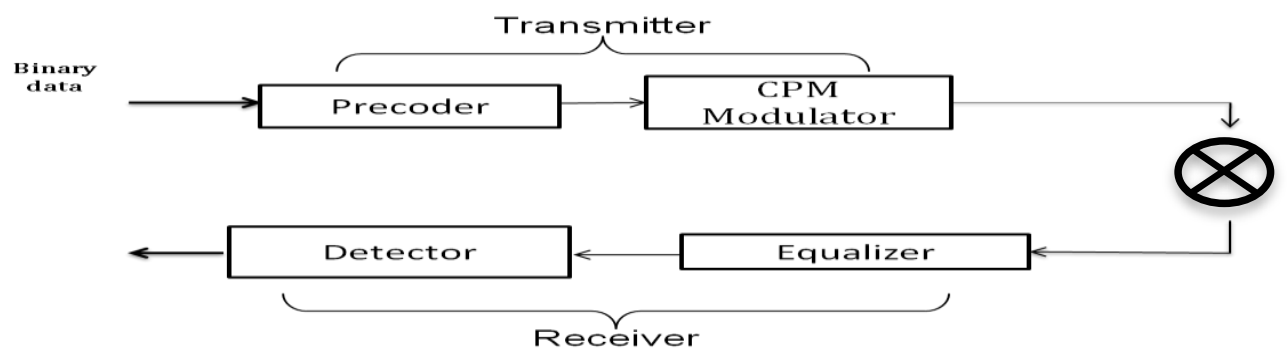

Figure 1: SOQPSK Communication System

As shown in Figure 1, the data is encoded using a precoder, which converts the binary input data to a ternary output stream consisting of $\{-1,0,+1\}$. The signal is then modulated using the CPM modulator with a pulse shape filter applied and this produces the SOQPSK signal which is transmitted over the channel. At the receiver inter-symbol interference (ISI) due to the channel effects is removed by equalization (CMA). Two block processing methods are discussed for processing the received data while updating the CMA equalizer. The equalizer output is then convolved with a detection filter, which acts like a matched filter and converts the equalized SOQPSK signal into QPSK form.

\section{CMA EQUALIZATION}

At the receiver, the CMA cost function is used to specify a blind equalizer which is based on the transmitted source signal. The cost function for our equalization scheme is the CMA cost function given in (1). The CMA cost function, $J_{C M A}(y)$, minimizes the distance of the equalizer output from a circle of known radius, which is normally determined by the known constellation points. The CMA cost function is given by

$$
J_{C M A}(y(n))=E\left\{\left(|y(n)|^{2}-R_{2}\right)^{2}\right\}
$$

where $R$ denotes the square value of the CMA equalizer radius and $y(\mathrm{n})$ represents the equalizer output block at time $\mathrm{n}$. It is known that SOQPSK modulation symbols of the transmitted modulated data are of a constant modulus and of radius 1, but in this work four different values of this CMA radius squared, $(R=1,2,3$ and 4$)$ are evaluated for equalization performance.

For CMA radius $R=1$, figure 2(a) and 2(b) shows respectively the equalized output and the QPSK 
constellation after the detection filter is applied at the receiver at $\mathrm{SNR}=18 \mathrm{~dB}$ for channel 1 .

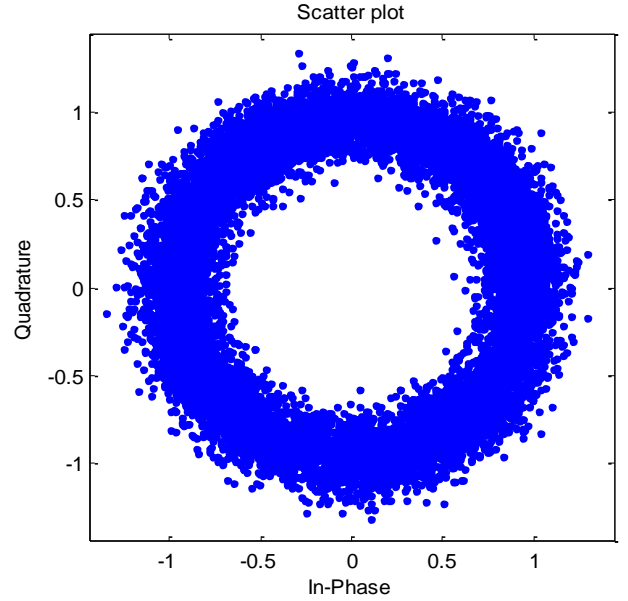

Figure 2(a): Equalized SOQPSK for radius1

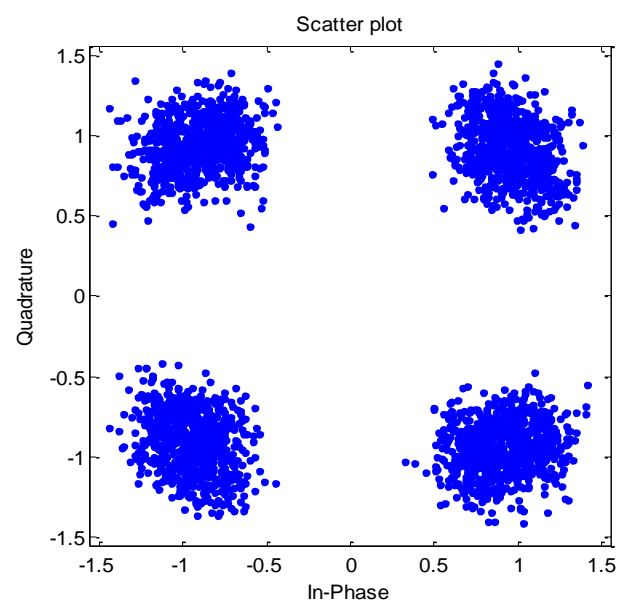

(b) Equalized QPSK constellation for radius 1

When a CMA radius squared value of $R=2$ is used, the equalized output and corresponding QPSK constellation respectively are shown in figures 3(a) and 3(b).

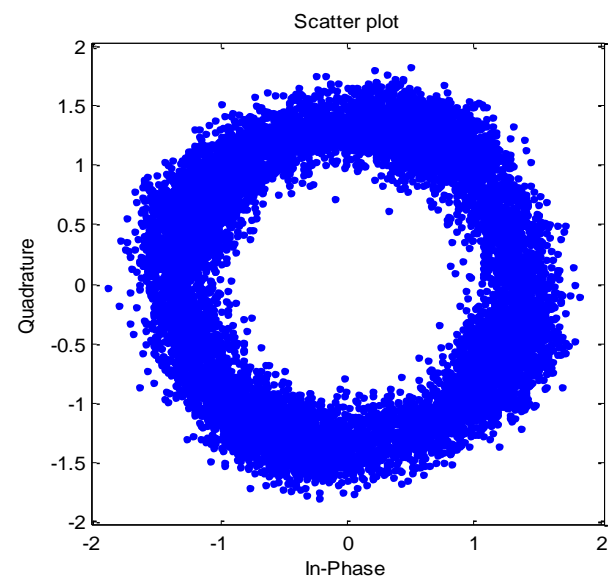

Figure 3: (a) Equalized output for radius 2

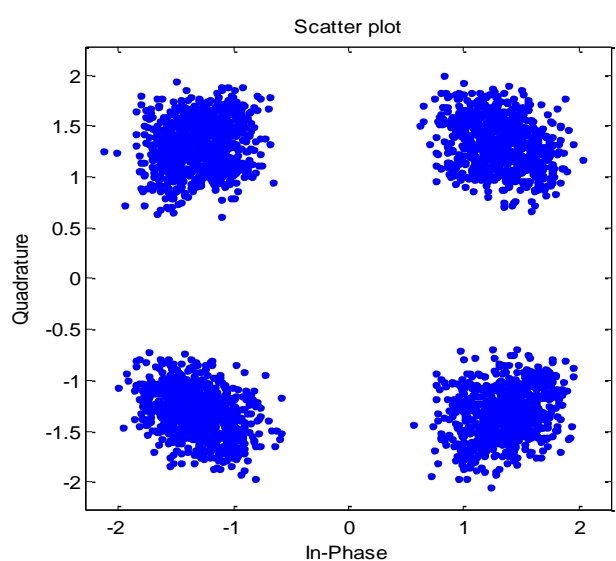

(b) Equalized QPSK constellation for radius 2

The symbols of the equalized SOQPSK modulated symbols appear to lie on a circle of radius $\sqrt{2}$ while the QPSK constellation points are located at $+/-\sqrt{2}$. Similar results have been observed for the higher values of radius, but for the radius squared values of $R=3$ and 4, the SOQPSK circle radius and constellation points actually lie at the values of 3 and 4 , respectively.

\section{IMPLEMENTATION OF CMA FOR AERONAUTICAL TELEMETRY}

In order to evaluate the equalizer performance, we will use the previously measured aeronautical telemetry channel 1 for simulation and testing [5]. This channel is of length 9 and was measured on the taxiway before the aircraft takes off. Flowcharts are presented below for the two real-time block processing methods implemented in this work. Section 4.1 describes block-by-block processing and section 4.2 describes single block processing. 


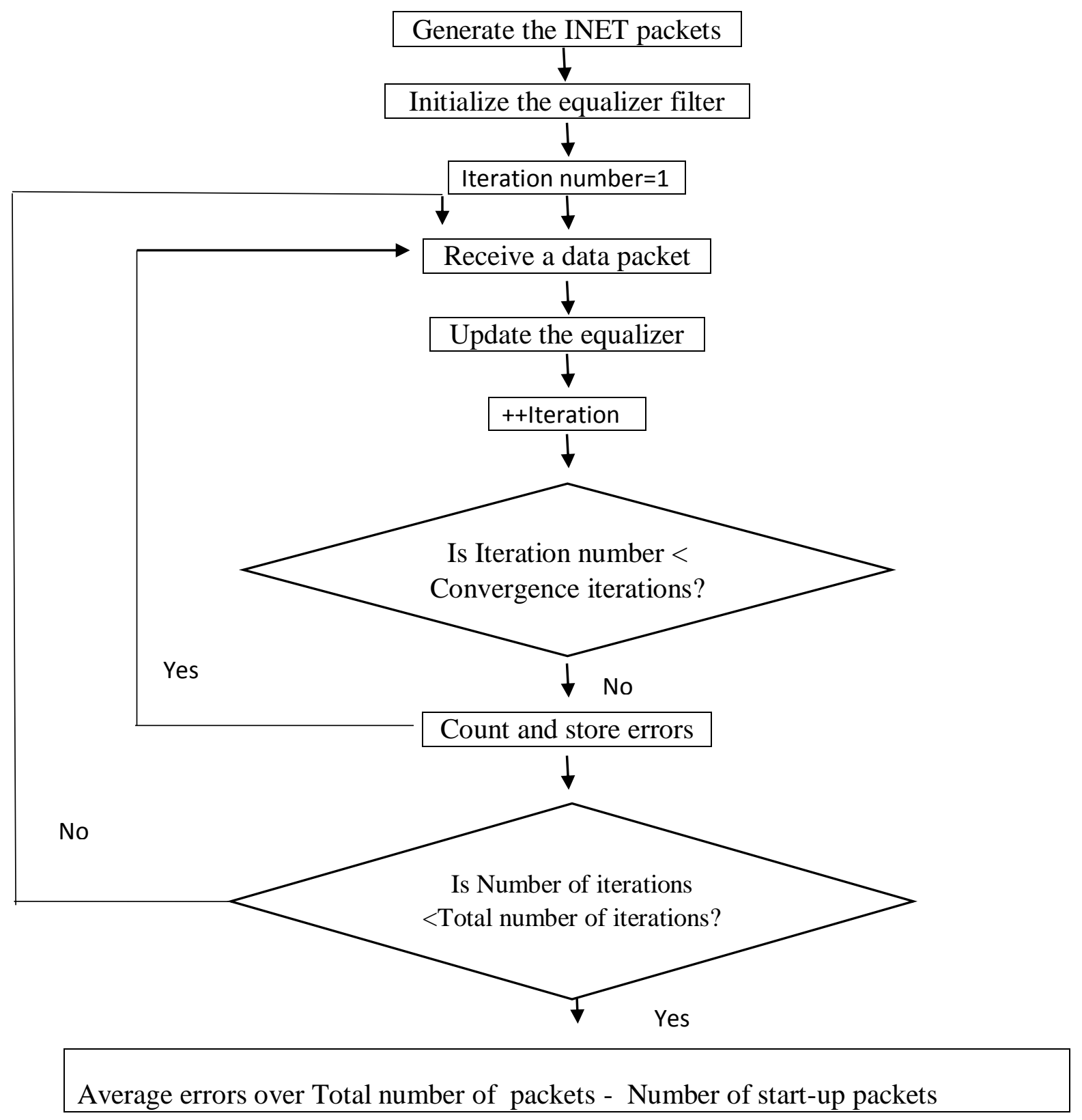

Figure 4: Flow chart of $\mathrm{BxB}$ processing

For $\mathrm{BxB}$ processing the $\mathrm{CMA}$ equalizer is updated iteratively on multiple packets. Errors are counted after a specified number of packets have been processed to allow for convergence, and averaging is done over a total number of specified packets. The flow chart in figure 4 shows the implementation of the block-by-block processing method. 


\subsection{Single Block Processing Method}

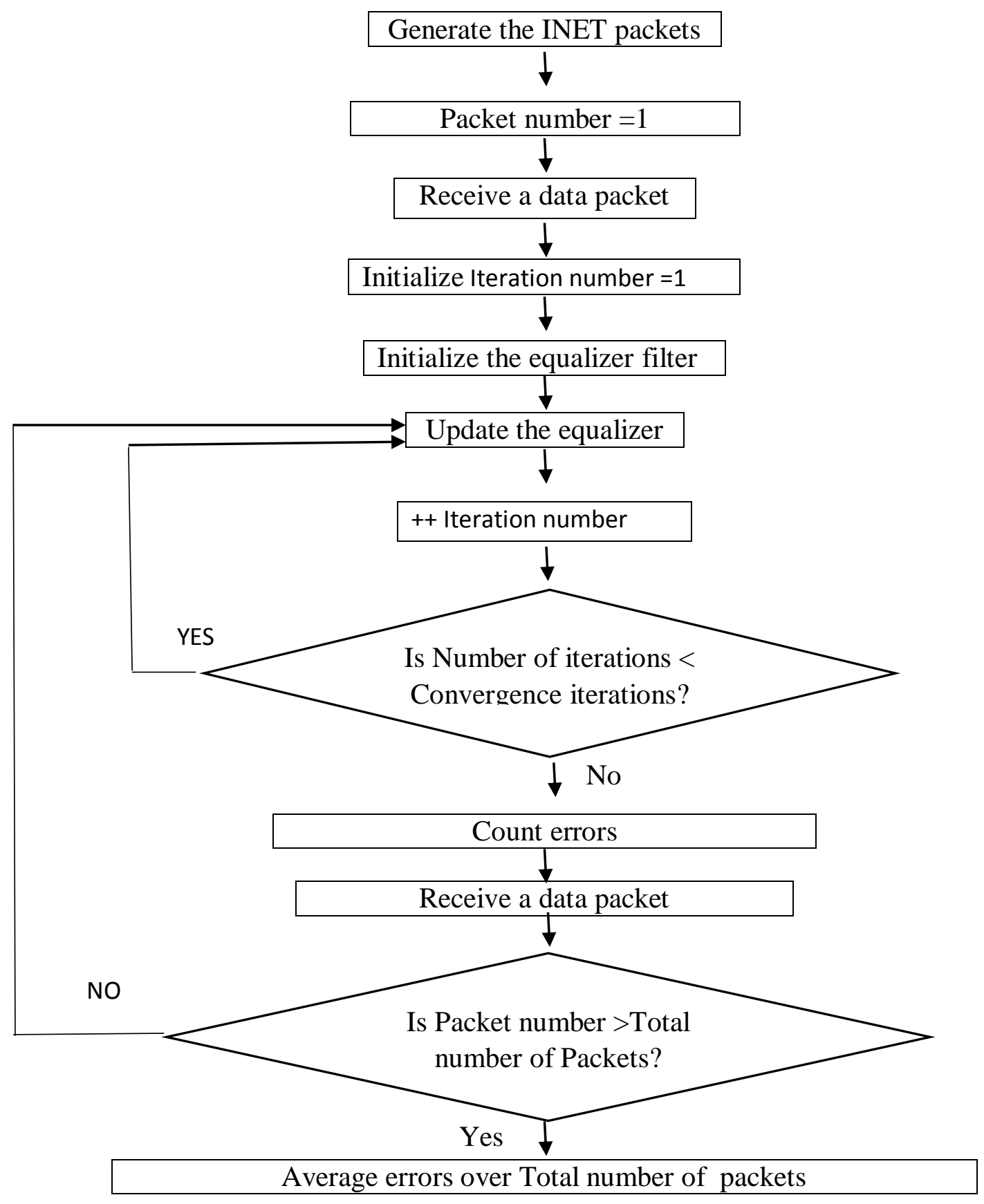

Figure 5: Flow chart of Single block processing

For single block processing the first packet is used to update the equalizer filter coefficients until convergence of the equalizer. The error analysis is then done by bringing in new packets to update the equalizer. 


\section{PERFORMANCE EVALUATION RESULTS}

In this section we present performance evaluation results for the CMA equalizer for channel 1. First the convergence curves for the block-by-block processing and single block processing methods are compared for different values of CMA radius in order to determine the number of packets required for initialization of each block processing method, and then we compare the bit error rate performance when using different values of CMA radius.

\subsection{Evaluation of different values of CMA radius}

In this section the values of CMA radius is changed and we compare the performance of the CMA equalizer by plotting the average bit error rate. First we consider the CMA cost function, $\mathrm{J}_{\mathrm{cma}}$ to determine the number of data packets required for convergence of the equalizer. Figures 6 (a) and (b) show for a single Monte Carlo run the convergence curves of $\mathrm{J}_{\mathrm{cma}}$ for $\mathrm{BxB}$ processing and for Single block processing respectively for radius squared values of $R=1,2,3$ and 4 .

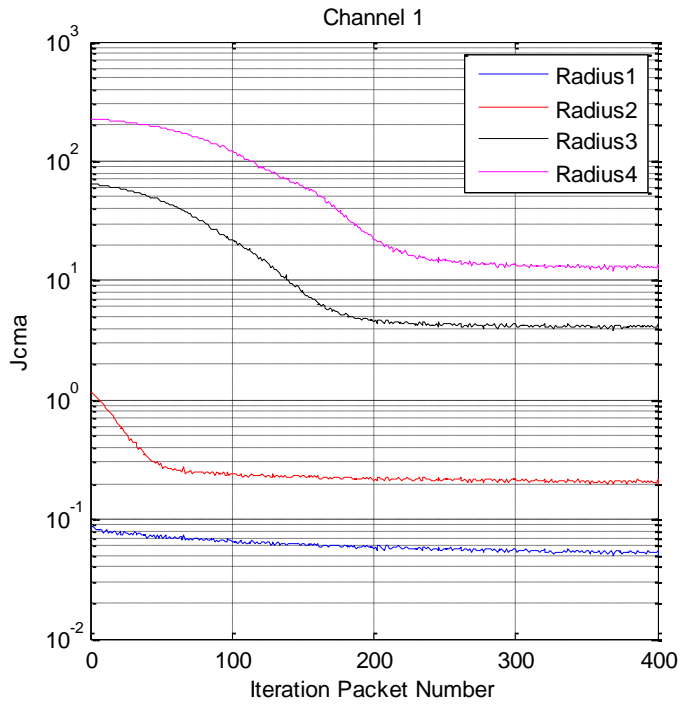

(a) Block-by-Block

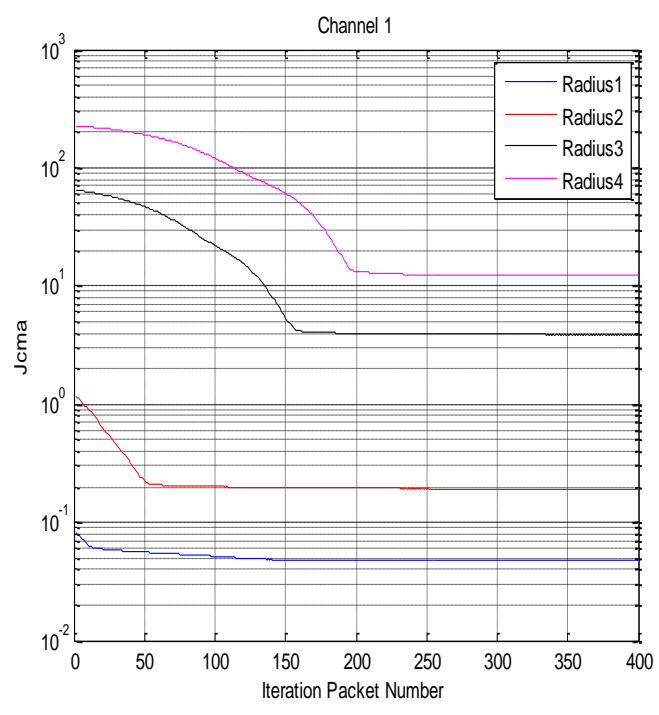

(b) Single Block

Figure 6: Convergence curves for $\mathrm{J}_{\mathrm{cma}}$ using radius, $R=1,2,3$, and 4

The number of data packets, which must be discarded during initialization for these two realtime methods, can be determined from the convergence curves of figures 6 (a) and (b). We note that the cost function value increases with increasing radius. In addition the larger the value of the radius the more iterations it takes for both methods to converge. 50 packets as startup (for convergence) appears to be sufficient when using a radius squared value of 1 and 2 for this particular Monte Carlo run, while radius 3 requires about 150 packets for convergence, and radius 4 requires about 250 packets for convergence for both methods. The number of packet iterations needed for initialization of the block-by-block processing method is comparable to the number of iterations required for the single block method. Table 1 summarizes the parameters that were chosen from the convergence curves of figure 6 , in order to perform the bit error analysis for the four different values of CMA radius. These results presented in figure 7 use the 
last column of Table 1 so that the number of packets used for error averaging is consistent for the two methods.

Table 1: Number of Packets used for Radius Performance Analysis

\begin{tabular}{|c|c|c|c|}
\hline Radius & $\begin{array}{c}\text { Total Block Length } \\
(\mathrm{BxB})\end{array}$ & $\begin{array}{c}\text { Convergence } \\
\text { iterations }\end{array}$ & Average errors over \\
\hline 1 & 700 & 50 & 650 \\
\hline 2 & 700 & 50 & 650 \\
\hline 3 & 750 & 150 & 600 \\
\hline 4 & 850 & 250 & 600 \\
\hline
\end{tabular}

In figure 7 the bit error rate curves for the two methods are plotted for channel 1 based on the packet initialization specified in Table 1 and determined from figures 6 . The theoretical lower bound curve for the AWGN channel is included to show the best achievable equalizer performance for a single tap channel with additive white Gaussian noise. Note that the best results are produced for CMA radius, $R=2$. A baseline curve for the performance of the MMSE CMA equalizer is also included. This curve was produced by taking a Monte Carlo average of the final error rate of an MMSE-initialized CMA equalizer where convergence is achieved using a single fixed packet [4]. It is not a real-time procedure and it produces almost identical results for both radius squared, $R=1$ and 2 .

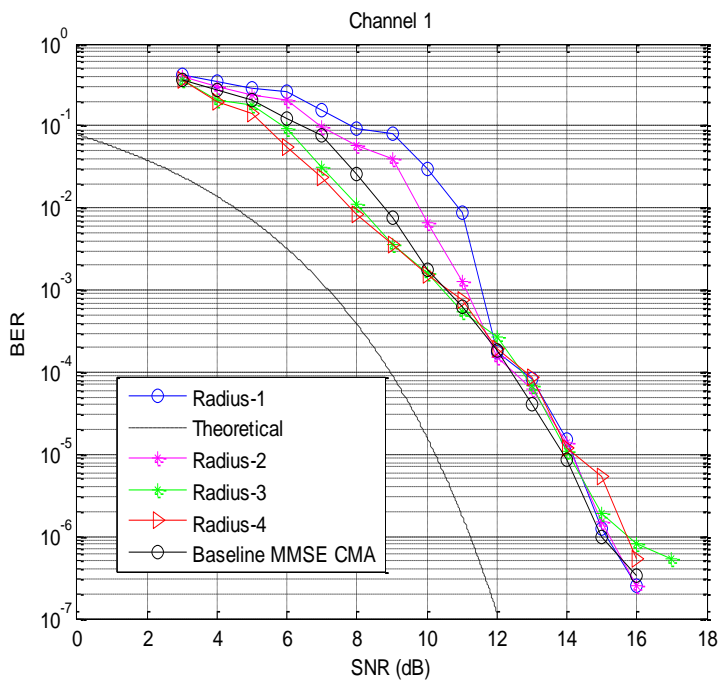

(a) Block-by-Block

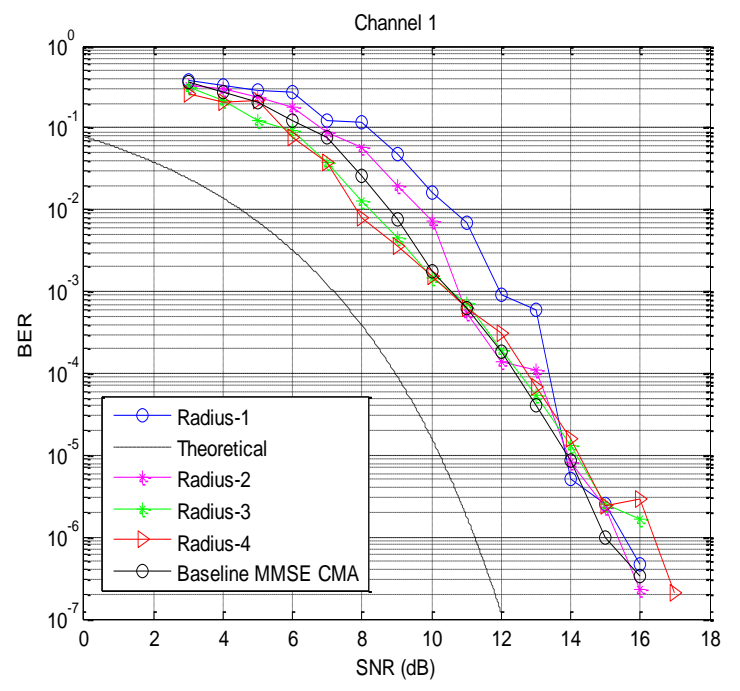

(b) Single Block

Figure 7: BER vs. SNR for different radius values $(R=1,2,3$, and 4) for channel 1.

We note from figure 7 that for both methods the radius $R=1$ curves produce the highest bit error rates at low SNR followed by radius squared, $R=2$, and both achieve the baseline performance of the MMSE CMA equalizer described above. At low SNRs, the radius, $R=3$ and 4 curves produce lower BER than even the baseline MMSE CMA, but at higher SNR their error rates are less stable and are higher than the baseline MMSE CMA. In addition radius $R=3$ and 4 require 
higher numbers of packets to be discarded during initialization when compared to radius, $R=1$ and 2. For the reasons stated above, we choose to continue the performance evaluation of CMA by comparing only radius, $R=1$ and 2 .

\subsection{Packet Length Comparison}

In this experiment, we test the effect of increasing the block length in BxB processing method for radius 1 and radius 2 using channel 1, by plotting bit error rate curves for different packet lengths. These results are shown in figure 8 .

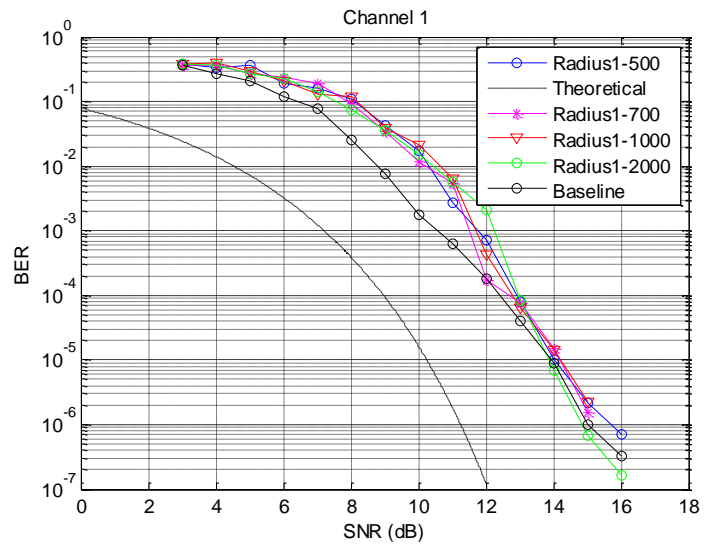

(a) Radius squared, $R=1$

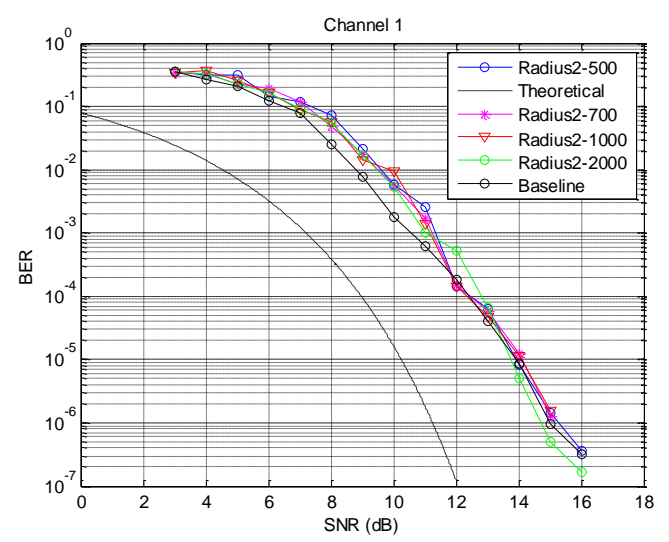

(b) Radius squared, $R=2$

Figure 8: Packet Length comparison for Block-by-block processing

CMA squared radius, $R=2$ continues to produce lower bit error rates than radius, $R=1$. The performance using radius squared, $R=2$ remains consistently stable as the packet length varies from 500 to 2000. For a packet length of 2000 we note improved performance at higher SNRs, except for a spike in errors which occurs at $12 \mathrm{~dB}$. The radius 1 curves are more varied for different packet lengths, but for a packet length of 2000 they also show improved performance at higher SNRs, with a similar spike in errors at $12 \mathrm{~dB}$. The Single block processing method has been observed to show similar performance to the BXB method for different packet lengths.

\subsection{BER Performance of $\mathrm{BxB}$ versus Single Block processing methods}

In this experiment we compare the block-by-block processing method with the single block processing method for the aeronautical telemetry channel 1 . Both block processing methods (with radius, $R=1$ and 2) will use an initial set of 50 packets for startup (and convergence) of the equalizer, so these packets are discarded. Errors are then counted and averaged over a total of 650 data packets for a total of 700 data packets. Figure 9 shows the error rates for the two realtime methods discussed here. 


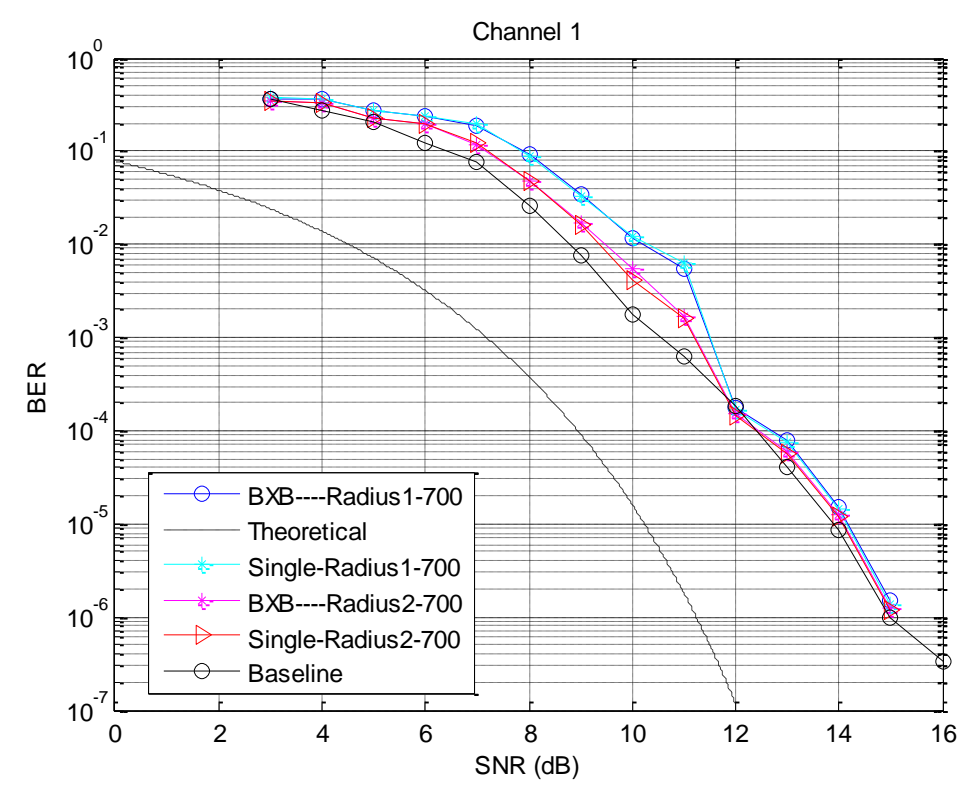

Figure 9: BER vs SNR for Channel 1 using radius squared, $R=1$ and 2

We observe that CMA radius squared, $R=2$ produces consistently better results for this channel. The performance of $\mathrm{BxB}$ method is very similar to that of single block method for radius, $R=2$, but there is more of a difference in the performance of the two methods when CMA radius 1 is used.

\section{CONCLUSIONS}

In this work, we investigated the effect of using different values of radius squared, $R$ for a CMA block equalizer for SOQPSK, which has been transmitted using the iNET data packet structure. The known data in the iNET packet structure is used to determine the MMSE equalizer which is used to initialize the CMA equalizer. Two implementations of the block processing CMA equalizer have been investigated, and these have been shown to have similar performance. Both of these methods are implemented to achieve real-time equalization of the transmitted data, and they both require that a number of data packets are discarded during convergence of the CMA equalizer.

In general, a CMA radius squared value of $R=2$ would be our best choice for the CMA radius, since it achieves consistently lower bit error rates over the full range of SNRs than radius 1. For the radius squared values of $R=3$ and 4 , the bit error rates at low SNR can be lower than that of radius squared $R=2$, but these require a larger number of data packets to be discarded for initialization and the high SNR behavior for these higher radius values appears to be unstable and it diverges from the baseline performance provided by the MMSE CMA equalizer curve.

\section{ACKNOWLEDGEMENTS}

This work was funded by the Test Resource Management Center (TRMC) Test and Evaluation Science and Technology (T\&E/S\&T) Program through the U.S. Army Program Executive Office for Simulation, Training and Instrumentation (PEO STRI) under contract W900KK_13-C-0026 (PAQ). Approved for public release; distribution is unlimited. 412TW-PA-14316. 


\section{REFERENCES}

[1] R. Johnson, et. al. "Blind Equalization using the Constant Modulus criterion: A Review", Proc, of IEEE, Vol.86, No.10, pp. 1927-1950, 1998.

[2] E. Law, "How well does a blind adaptive CMA equalizer work in a simulated telemetry multipath environment", Proceedings of the International Telemetry Conference (ITC) 2004, San Diego, CA.

[3] A.Cole-Rhodes, H. Umuolo, F. Moazzami, M. Rice, "Real-Time CMA Equalization of SOQPSK for Aeronautical Telemetry", submitted to ITC 2014.

[4] A.Cole-Rhodes, H. Umuolo, F. Moazzami, M. Rice, "A Block Processing Approach to CMA Equalization of SOQPSK for Aeronautical Telemetry”, submitted to MILCOM 2014.

[5] M. Rice, "Phase 1 Report: Preamble Assisted Equalization for aeronautical telemetry (PAQ)," Brigham Young University, Technical Report, 2014, submitted to the Spectrum Efficient Technologies (SET) Office of the Science \& Technology, Test \& Evaluation (S\&T/T\&E) Program, Test Resource Management Center (TRMC). Also available on-line at http://hdl.lib.byu.edu/1877/3242. 